\title{
ORIGINAL
}

\section{FACTORES ASOCIADOS AL CUMPLIMIENTO DE LAS RECOMENDACIONES SOBRE ACTIVIDAD FÍSICA EN POBLACIÓN TRABAJADORA DE ENTRE 40 Y 55 AÑOS DE EDAD}

\section{Belén Moreno-Franco (1, 2), José L Peñalvo (3), José A Casasnovas Lenguas (1,2) y Montserrat Leon-Latre (1).}

(1) Unidad de Prevención Cardiovascular. Instituto Aragonés de Ciencias de la Salud (IACS). Fundación Instituto de Investigación Sanitaria de Aragón (IIS Aragón). Zaragoza.

(2) Departamento de Medicina, Psiquiatría y Dermatología. Universidad de Zaragoza. Zaragoza.

(3) School of Nutrition Science and Policy. Tufts University. Boston, Massachusetts.

(*) Este estudio ha sido financiado en parte por el Fondo de Investigaciones Sanitarias FIS PI11/00403, PI12/01434 y soportado estructuralmente por convenio de colaboración entre el Departamento de Salud del Gobierno de Aragón y el Centro Nacional de Investigaciones Cardiovasculares del Instituto Carlos III.

Los autores declaran que no existe conflicto de intereses en la realización del estudio.

\section{RESUMEN}

Fundamentos: La inactividad física continúa siendo un problema de salud pública a nivel mundial. El objetivo del presente estudio fue determinar la adherencia a las recomendaciones de actividad física (AF) establecidas por la Organización Mundial de la Salud (OMS) de una muestra de trabajadores españoles, así como analizar su asociación con diferentes factores de riesgo cardiovascular (FRCV), indicadores sociodemográficos y de estilo de vida.

Métodos: Estudio tranversal con 2.651 participantes en la cohorte del Aragon Workers' Health Study (AWHS) (2.428 varones y 133 mujeres), con edades comprendidas entre los 40-55 años. Se dividió a la población en dos grupos en función de si habían cumplido o no durante el último año las recomendaciones sobre AF establecidas por la OMS, teniendo en cuenta las actividades relacionadas con la ocupación, ocio y recreación y desplazamientos. Se registraron variables sociodemográficas, antropométricas, clínicas, farmacológicas y de estilo de vida entre los años 2011-2014. Se calcularon la mediana y el intervalo intercuartílico para variables cuantitativas y la distribución de frecuencias para las categóricas. Se estimó un modelo de regresión logística binaria crudo y ajustado para analizar la probabilidad de cumplir las recomendaciones de $\mathrm{AF}$ en función del número de FRCV (hipertensión, dislipemia, diabetes, obesidad y tabaquismo).

Resultados: El 47,0\% de participantes cumplían las recomendaciones. El cumplimiento fue del $39,7 \%$ en hipertensos, del $38,7 \%$ en dislipémicos y $32,7 \%$ en diabéticos. El 51,3\% reportó no realizar AF o realizar una AF ligera, el $20,2 \%$ moderada y el $28,5 \%$ vigorosa o muy vigorosa. Los resultados mostraron cómo a medida que aumentaba el número de FRCV disminuía la probabilidad de adherirse a las recomendaciones: 1 FRCV (OR: 0,62; 95\%CI: 0,49-0,78), 2 FRCV (OR: 0,49; 95\%CI: 0,38-0,62), 3 FRCV (OR: 0,34; 95\%CI: 0,25-0,46), 4 FRCV (OR: 0,19; 95\%CI: 0,11-0,32).

Conclusiones: El incumplimiento de las recomendaciones de $\mathrm{AF}$ en nuestra población es frecuente sobre todo en personas con mayor riesgo cardiovascular.

Palabras clave: Actividad física. Recomendaciones. Cumplimiento. Estilo de vida. Enfermedad cardiovascular.

\section{Correspondencia}

Belén Moreno-Franco

Unidad de Prevención Cardiovascular

Instituto Aragonés de Ciencias de la Salud (IACS)

Fundación Instituto de Investigación Sanitaria Aragón (IIS Aragón)

Hospital Universitario Miguel Servet

C/Isabel La Católica 1-3, Edif. Principal, Planta +2

50009 Zaragoza, España

bmorenof.iacs@aragon.es

\section{ABSTRACT \\ Compliance with Physical Activity Recommendations and Associated Factors in a Cohort of Spanish Adult Workers}

Background: Physical inactivity remains a public health problem worldwide. The purpose of this study was to determine compliance with physical activity (PA) recommendations developed by the World Health Organization (WHO) and to analyze the association with different cardiovascular risk factors (CVRF) and sociodemographic and lifestyle determinants in a sample of Spanish workers.

Methods: A cross sectional analysis was carried out in a sample of 2.651 individuals ( 2.428 men and 133 women, aged 40-55 years old) from the Aragon Workers' Health Study (AWHS). Sample was divided into two groups depending on whether they met PA recommendations during the last year, including transportation, leisure and occupational PA. Sociodemographic, anthropometric, clinical, pharmacological and lifestyle data were collected during the years 2011-2014. Median and interquartile range for quantitative variables and frequency distribution for categorical variables were used. Crude and adjusted binary logistic regression models were carried out to study the likelihood of meeting with PA recommendations based on the number of CVRF (hypertension, dyslipidemia, diabetes, obesity and smoking) present.

Results: $47.0 \%$ of workers complied with WHO recommendations for PA. Lower prevalence of compliance was found among those participants presenting some CVRF (39.7\% in hypertensive, $38.7 \%$ in dyslipidemic and $32.7 \%$ in diabetic). Of the total study sample, $51.3 \%$ reported not performing PA or practice a light PA, $20.2 \%$ moderate and $28.5 \%$ vigorous or very vigorous PA. The likelihood of compliance with PA recommendations decreased with increasing the number of CVRF: 1 CVRF (OR: $0.62 ; 95 \% \mathrm{IC}$ : 0.49-0.78), 2 CVRF (OR: 0.49; 95\% IC: 0.38-0.62), 3 CVRF (OR: 0.34 ; 95\% IC: 0.25-0.46), 4 CVRF (OR: 0.19; 95\% IC: 0.11-0.32).

Conclusions: Compliance with recommendations of PA in our population remains insufficient, moreover in those with increased cardiovascular risk.

Keywords: Physical activity. Recommendations. Compliance. Lifestyle. Cardiovascular disease.

DOI: 


\section{INTRODUCCIÓN}

Los beneficios de la práctica de actividad física (AF) son ampliamente conocidos por la mayor parte de la población y han sido abordados en múltiples guías con indicaciones detalladas sobre cantidad, frecuencia e intensidad de AF a realizar ${ }^{1-3}$. A pesar de ello, un amplio porcentaje de la población mundial sigue siendo físicamente inactiva, lo que constituye el cuarto factor de riesgo de mortalidad más importante responsable del $6,0 \%$ de las defunciones anuales en todo el mundo ${ }^{4}$. La población adulta se enfrenta, en su mayoría, a una reducción significativa en la exigencia física en su lugar de trabajo y durante el tiempo de ocio muy pocas personas son físicamente activas. Esta situación no puede atribuirse exclusivamente a la motivación personal sino a un complejo entorno laboral, social y familiar. Es por ello que los países que abordan este tema complejo optan cada vez más por utilizar enfoques multidisciplinares para intentar incrementar la práctica de AF en la población. No obstante, las acciones comunitarias dirigidas a este fin, sobretodo en atención primaria, no muestran todavía un aumento efectivo y mantenido de los niveles de $\mathrm{AF}^{5}$.

La Organización Mundial de la Salud (OMS) en su informe del año 2010 presentó sus recomendaciones mundiales sobre AF para la salud ${ }^{1}$ con el objetivo de prevenir las enfermedades no transmisibles. Consistentes en la práctica de al menos 150 minutos semanales de AF aeróbica moderada o bien 75 minutos semanales de AF aeróbica vigorosa o una combinación de ambas siempre en sesiones de al menos 10 minutos, estas recomendaciones, pueden ser aplicadas también a personas con enfermedades crónicas no relacionadas con la movilidad, como son la hipertensión, la diabetes mellitus o cualquier tipo de dislipemia, ya que se asocian con un mayor riesgo de sufrir un evento isquémico.

El objetivo del estudio fue definir el grado de adherencia a las recomendaciones de AF propuestas por la OMS en un grupo de trabajadores de la cohorte del Aragon Workers
Health Study (AWHS) ${ }^{6}$, así como analizar su asociación con diferentes factores de riesgo cardiovascular (FRCV), indicadores sociodemográficos y de estilo de vida.

\section{SUJETOS Y MÉTODO}

Diseño y población de estudio. El análisis se llevó a cabo sobre una muestra de población del estudio AWHS, cuyo diseño y metodología han sido previamente publicados ${ }^{6}$. Se trata de un estudio de cohorte prospectivo basado en los exámenes de salud de los trabajadores de la factoría de General Motors España. Su objetivo principal es determinar los condicionantes genéticos y de estilo de vida que determinan la aparición y progresión de FRCV. Cada año, aproximadamente un tercio de los 2.978 trabajadores con edades comprendidas entre los 40 y 55 años, son seleccionados para llevar a cabo exámenes complementarios de aterosclerosis subclínica por imagen básica, la cumplimentación de cuestionarios de dieta, AF y estilos de vida así como una anamnesis clínica.

Para el presente estudio se realizó un análisis transversal de los primeros 2.561 voluntarios estudiados durante los tres primeros años, que representan el $86,0 \%$ de los trabajadores de la factoría en ese intervalo de edad. El estudio fue aprobado por el Comité Ético de Investigación Clínica de Aragón. Asimismo se obtuvo el consentimiento informado de todos los participantes.

Valoración de actividad física y estilo de vida. Para la valoración del cumplimiento de las recomendaciones de AF se utilizó la versión española validada del cuestionario de práctica de actividades utilizado en el Nurses' Health Study y el Health Professionals' Follow-up Study ${ }^{7}$. Se dividió a la población en dos grupos en función de si habían cumplido o no durante el último año las recomendaciones de AF establecidas por la $\mathrm{OMS}^{1}$. Se consideró que las cumplían quienes reportaron una práctica de 150 minutos semanales de AF aeróbica moderada o bien 75 minutos semanales de AF aeróbica vigorosa o bien 
una combinación de ambas en sesiones de al menos 10 minutos. Los participantes debían indicar en una escala de 0 a 10 el grado de intensidad en el esfuerzo, considerando de 0 a 4 como actividad ligera, de 5 a 6 como moderada, de 7 a 8 como vigorosa y de 9 a 10 como actividad muy vigorosa. Según las directrices de la OMS, se tuvieron en cuenta las actividades recreativas o de ocio, desplazamientos (por ejemplo paseos en pie o en bicicleta), actividades laborales, tareas domésticas, juegos, deportes o ejercicios programados en el contexto de las actividades diarias, familiares y comunitarias. Para valorar los periodos invertidos en actividades sedentarias se preguntó acerca del tiempo dedicado tanto en días laborables como de fin de semana a: horas de sueño (h/día), horas de siesta (h/día), tiempo de televisión (h/día) y tiempo sentado (h/día). Los participantes completaron además un cuestionario adicional sobre características sociodemográficas que comprendía preguntas sobre edad, sexo, nivel educativo, turno y tipo de trabajo.

La evaluación de los hábitos alimentarios se realizó mediante un cuestionario semicuantitativo de frecuencia de consumo de alimentos (CFCA) validado previamente para la población española ${ }^{8,9}$, con preguntas acerca de la ingesta media de 136 alimentos durante el último año. Los datos fueron posteriormente transformados en nutrientes mediante tablas españolas de composición de alimentos $^{10,11}$. Para estudiar la adherencia al patrón de dieta mediterránea se utilizó el índice de calidad de dieta alternate Mediterranean Diet Index (aMED) $)^{12}$, que evalúa la ingesta de nueve componentes, asignando un rango de puntuación de 0 (menor adherencia) a 9 (mayor adherencia).

Variables clínicas y antropométricas. La medida de la presión arterial se llevó a cabo tras reposo físico de al menos cinco minutos con un oscilómetro digital OMRON M10-IT (OMRON Healthcare Co Ltd; Japan) calculándose el promedio de tres lecturas consecutivas. Se midieron peso, talla y perímetro de cintura y se calculó el índice de masa corporal (IMC). Los sujetos aportaron además información referente a su historia clínica, incluyendo antecedentes familiares o personales de enfermedad cardiovascular precoz (ECV), toma de medicamentos o el diagnóstico previo de hipertensión arterial, diabetes mellitus o dislipemia. Las determinaciones de glucosa, colesterol total, triglicéridos y colesterol unido a lipoproteínas de alta densidad (cHDL) se llevaron a cabo mediante análisis enzimáticos con el equipo ILab 650 de Instrumentation Laboratory. Las concentraciones de apolipoproteina A1, apolipoproteina B100 y proteína $\mathrm{C}$ reactiva (PCR) se determinaron por nefelometría con el equipo IMMAGE 800 de Beckman Coulter. La insulina ultrasensible se determinó mediante inmunoanálisis de quimioluminiscencia con el equipo Access de Beckman Coulter. La hemoglobina glicosilada (HbAlc) se determinó mediante intercambio de cationes en columna de fase inversa usando el equipo ADAMS A1c HA-810 de Arkray Factory. Las concentraciones de colesterol unido a lipoproteínas de baja densidad (cLDL) se calcularon usando la fórmula de Friedewald cuando los valores de triglicéridos eran $<400 \mathrm{mg} / \mathrm{dL}^{13}$. Además se calculó el índice de resistencia insulínica homeostasis model assessment of insulin resistance (HOMA-IR) como medida de determinación de resistencia a insulina ${ }^{14}$.

Definición de factores de riesgo cardiovascular. Para el diagnóstico de hipertensión arterial se definió un valor de presión arterial superior a 140/90 mmHg y/o tratamiento antihipertensivo en curso. La dislipemia se definió como concentraciones de colesterol total $\geq 240 \mathrm{mg} / \mathrm{dL}$ y/o cLDL $\geq 160 \mathrm{mg} / \mathrm{dL}$ y/o cHDL $<40 \mathrm{mg} / \mathrm{dL}$ en hombres o cHDL $<50 \mathrm{mg} / \mathrm{dL}$ en mujeres y/o tratamiento hipolipemiante en curso. Se consideró diabéticas a las personas con niveles de glucosa en ayunas $\geq 126 \mathrm{mg} / \mathrm{dL}$ en al menos una determinación y/o diagnóstico de diabetes en la historia clínica previa y/o toma de medicación antidiabética. La obesidad se definió como los valores de IMC $\geq 30$ $\mathrm{kg} / \mathrm{m}^{2}$. Se consideró fumador a quien fumaba 
en el momento del examen o si había transcurrido menos de un año desde el abandono tabáquico, exfumador a quien había consumido al menos 50 cigarrillos a lo largo de su vida y había transcurrido más de un año desde el consumo del último cigarrillo y no fumadores a los que no habían fumado nunca o habían fumado menos de 50 cigarrillos.

Análisis estadístico. Se comprobó la normalidad de las variables mediante el test de Kolmogorov-Smirnov. Se calculó el valor de la mediana y su intervalo intercuartílico (IIC) para variables cuantitativas y la distribución de frecuencias para las categóricas. Las diferencias entre grupos fueron analizadas mediante el contraste no paramétrico U de Mann-Whitney para variables cuantitativas y la prueba de la chi $^{2}$ en el caso de las categóricas. Se llevó a cabo un análisis multivariante mediante regresión logística binaria en el que la variable dependiente fue cumplir o no las recomendaciones de AF y la variable independiente el número de FRCV, siendo la ausencia de FRCV el valor de referencia. Se calcularon los valores crudos y ajustados por edad y sexo (modelo 1) y adicionalmente ajustado por nivel educativo y turno de trabajo (modelo 2). Los resultados se presentan como odds ratios (OR) crudas y ajustadas junto con su intervalo de confianza (IC) del 95\%. Para el análisis se utilizó el programa IBM SPSS Statistics 22.0, considerando todos los contrastes a dos colas y un nivel de significación estadística del 5\%.

\section{RESULTADOS}

La muestra estuvo formada por 2.561 trabajadores (2.428 varones y 133 mujeres) cuya mediana de edad (ICC) fue de 52,2 (49,0-54,8) años. De ellos 1.204 (47,0\%) cumplían las recomendaciones de AF y 1.357 (53,0\%) declararon no hacerlo. El 51,3\% reportaron no realizar $\mathrm{AF}$ o realizar una $\mathrm{AF}$ ligera, el 20,2\% moderada y el 28,5\% vigorosa o muy vigorosa. Las actividades que más minutos aportaron al cumplimiento de las recomendaciones fueron andar o pasear fuera de casa y pasear en bicicleta, con una práctica media de 180,1 y 97,5 minutos a la semana respectivamente.

La tabla 1 describe la frecuencia del cumplimiento de las recomendaciones en función de las características sociodemográficas de la población objeto de estudio. La adherencia a las recomendaciones fue del 53,9\% en los participantes con edades comprendidas entre los 40-45 años, del 47,7\% en los correspondientes al rango de 46-50 años y del $45,6 \%$ en los incluidos en el grupo de edad de 51-55 años $(p=0,028)$.

Con respecto al nivel educativo se observó una prevalencia del $56,1 \%$ en quienes tenían estudios universitarios, del $48,2 \%$ en los que habían realizado formación profesional, del $50 \%$ en quienes tenían bachillerato y $44,3 \%$ en los que poseían estudios primarios $(\mathrm{p}=0,017)$.

La adherencia en función del turno de trabajo fue del $61,1 \%$ en el turno fijo diurno, $48,0 \%$ en el turno fijo nocturno y $44,9 \%$ en el turno rotatorio $(p<0,001)$. En relación al tipo de trabajo desempeñado en la empresa, la prevalencia fue del $45,6 \%$ en quienes realizaban trabajos manuales y del $55,6 \%$ en los que realizaban trabajo de oficina $(\mathrm{p}<0,001)$.

El perfil clínico y bioquímico de los trabajadores se presenta descrito en la tabla 2, donde la prevalencia en el cumplimiento de las recomendaciones fue del $51,9 \%$ en los sujetos con normo peso; del $49,6 \%$ en quienes tenían sobrepeso y del $36,1 \%$ en los que presentaban obesidad $(p<0,001)$. En relación a la medida de la circunferencia de la cintura se encontraron diferencias estadísticamente significativas entre el grupo que cumplía las recomendaciones y el que no las cumplía (98,5 vs. $95,5 \mathrm{~cm})(p<0,001)$. No se observaron diferencias estadísticamente significativas respecto a la presión arterial.

Con respecto a los lípidos plasmáticos, se encontraron diferencias estadísticamente significativas entre ambos grupos en los niveles de triglicéridos (133,0 vs 117,0 mg/ 


\begin{tabular}{|c|c|c|c|c|}
\hline \multicolumn{5}{|c|}{\begin{tabular}{|c|} 
Tabla 1 \\
$\begin{array}{c}\text { Características sociodemográficas de la población de estudio } \\
\text { en función de la adherencia a las recomendaciones de actividad física } \\
\text { establecidas por la Organización Mundial de la Salud }\end{array}$ \\
\end{tabular}} \\
\hline \multirow{2}{*}{\multicolumn{2}{|c|}{ Variables }} & \multicolumn{2}{|c|}{$\begin{array}{c}\text { Adherencia } \\
\text { a las recomendaciones de } \mathrm{AF}\end{array}$} & \multirow[b]{2}{*}{$\mathrm{p}$} \\
\hline & & \multirow{2}{*}{$\begin{array}{c}\text { No } \\
1.357(53,0) \\
53,2 \\
\end{array}$} & \multirow{2}{*}{\begin{tabular}{|c|} 
Sí \\
$1.204(47,0)$ \\
46,8 \\
\end{tabular}} & \\
\hline Sexo & Varones & & & \multirow{2}{*}{0,425} \\
\hline$(\%)$ & Mujeres & 49,6 & 50,4 & \\
\hline \multirow{3}{*}{$\begin{array}{c}\text { Edad } \\
(\%)\end{array}$} & $40-45$ & 46,1 & 53,9 & \multirow{3}{*}{0,028} \\
\hline & $46-50$ & 52,3 & 47,7 & \\
\hline & $51-55$ & 54,4 & 45,6 & \\
\hline \multirow{4}{*}{$\begin{array}{l}\text { Nivel educativo } \\
(\%)\end{array}$} & Estudios primarios & 55,7 & 44,3 & \multirow{4}{*}{0,017} \\
\hline & Bachillerato & 50,0 & 50,0 & \\
\hline & Formación profesional & 51,8 & 48,2 & \\
\hline & Estudios universitarios & 43,9 & 56,1 & \\
\hline \multirow{2}{*}{$\begin{array}{c}\text { Tipo de trabajo } \\
(\%)\end{array}$} & Manual & 54,4 & 45,6 & \multirow{2}{*}{$<0,001$} \\
\hline & Oficina & 44,4 & 55,6 & \\
\hline \multirow{3}{*}{$\begin{array}{l}\text { Turno de trabajo } \\
\qquad(\%)\end{array}$} & Fijo diurno & 38,9 & 61,1 & \multirow{3}{*}{$<0,001$} \\
\hline & Fijo nocturno & 52,0 & 48,0 & \\
\hline & Rotatorio & 55,1 & 44,9 & \\
\hline
\end{tabular}

\begin{tabular}{|c|c|c|c|c|c|}
\hline \multicolumn{6}{|c|}{$\begin{array}{l}\text { Tabla } 2 \\
\text { Perfil clínico y bioquímico de la población de estudio en función de la adherencia } \\
\text { a las recomendaciones de AF establecidas por la Organización Mundial de la Salud }\end{array}$} \\
\hline \multirow[t]{2}{*}{ Variables } & \multicolumn{4}{|c|}{$\begin{array}{c}\text { Adherencia } \\
\text { a las recomendaciones de actividad física }\end{array}$} & \multirow{2}{*}{$\mathrm{p}$} \\
\hline & & & No $1.357(53,0 \%)$ & Sí $1.204(47,0 \%)$ & \\
\hline \multirow{6}{*}{$\begin{array}{l}\text { Antropometría } \\
\text { y presión arterial }\end{array}$} & \multirow{3}{*}{$\begin{array}{c}\text { Indice } \\
\text { de masa corporal } \\
\left(\mathrm{Kg} / \mathrm{m}^{2}\right)\end{array}$} & Normo peso & 48,1 & 51,9 & \multirow{3}{*}{$<0,001$} \\
\hline & & Sobrepeso & 50,4 & 49,6 & \\
\hline & & Obesidad & 63,9 & 36,1 & \\
\hline & \multicolumn{2}{|c|}{ Circunferencia de cintura (cm) } & $98,5(92,5-104,9)$ & $95,5(89,5-101,3)$ & $<0,001$ \\
\hline & \multicolumn{2}{|c|}{ Presión arterial sistólica $(\mathrm{mmHg})$} & $124,0(115,0-134,0)$ & $124,0(115,0-133,8)$ & 0,841 \\
\hline & \multicolumn{2}{|c|}{ Presión arterial diastólica $(\mathrm{mmHg})$} & $83,0(76,0-89,0)$ & $82,0(76,0-88,0)$ & 0,077 \\
\hline \multirow{6}{*}{$\begin{array}{l}\text { Lípidos } \\
\text { plasmáticos } \\
(\mathrm{mg} / \mathrm{dL})\end{array}$} & \multicolumn{2}{|l|}{ Colesterol total } & $219,0(194,3-242,0)$ & $220,0(197,0-245,0)$ & 0,319 \\
\hline & \multicolumn{2}{|l|}{\begin{tabular}{|l|} 
Triglicéridos \\
\end{tabular}} & $133,0(91,0-196,0)$ & $117,0(82,0-169,0)$ & $<0,001$ \\
\hline & \multicolumn{2}{|l|}{ cHDL } & $51,0(44,0-58,0)$ & $54,0(47,0-62,0)$ & $<0,001$ \\
\hline & \multicolumn{2}{|l|}{ cLDL } & $136,1(115,7-157,0)$ & $136,8(118,0-158,0)$ & 0,320 \\
\hline & \multicolumn{2}{|c|}{ Apolipoproteina A1 } & $140,0(129,0-154,0)$ & $146,0(135,0-159,0)$ & $<0,001$ \\
\hline & \multicolumn{2}{|c|}{ Apolipoproteina B100 } & $106,0(91,0-121,0)$ & $102,0(88,0-119,0)$ & 0,024 \\
\hline \multirow{4}{*}{$\begin{array}{l}\text { Resistencia } \\
\text { a la insulina }\end{array}$} & \multicolumn{2}{|l|}{ Glucosa $(\mathrm{mg} / \mathrm{dL})$} & $96,0(89,0-104,0)$ & $95,0(88,0-102,0)$ & 0,003 \\
\hline & \multicolumn{2}{|l|}{ HbAlc (\%) } & $5,5(5,3-5,7)$ & $5,4(5,3-5,6)$ & $<0,001$ \\
\hline & \multicolumn{2}{|l|}{ Insulina (uU/mL) } & $7,0(5,0-10,1)$ & $5,6(4,1-8,2)$ & $<0,001$ \\
\hline & \multicolumn{2}{|l|}{ HOMA-IR } & $1,68(1,16-2,49)$ & $1,33(0,96-1,99)$ & $<0,001$ \\
\hline Inflamación & \multicolumn{2}{|c|}{ Proteína $\mathrm{C}$ reactiva $(\mathrm{mg} / \mathrm{dL})$} & $0,22(0,11-0,38)$ & $0,16(0,08-0,32)$ & $<0,001$ \\
\hline \multirow{3}{*}{ Tratamientos (\%) } & \multicolumn{2}{|c|}{ Tratamiento para hipertensión } & 60,3 & 39,7 & $<0,001$ \\
\hline & \multicolumn{2}{|c|}{ Tratamiento para dislipemia } & 61,3 & 38,7 & $<0,001$ \\
\hline & \multicolumn{2}{|c|}{ Tratamiento para diabetes mellitus } & 67,3 & 32,7 & 0,004 \\
\hline
\end{tabular}




\begin{tabular}{|c|c|c|c|c|}
\hline \multicolumn{5}{|c|}{$\begin{array}{c}\text { Tabla } 3 \\
\text { Características del estilo de vida que se asocian a la adherencia } \\
\text { de las recomendaciones de AF establecidas por la Organización Mundial de la Salud }\end{array}$} \\
\hline \multirow{2}{*}{ Variables } & \multicolumn{3}{|c|}{$\begin{array}{c}\text { Adherencia } \\
\text { a las recomendaciones de AF }\end{array}$} & \multirow{2}{*}{$\mathrm{p}$} \\
\hline & & $\begin{array}{c}\text { No } \\
1.357(53,0 \%)\end{array}$ & $\begin{array}{c}\text { Sí } \\
1.204(47,0 \%)\end{array}$ & \\
\hline \multirow{3}{*}{$\begin{array}{l}\text { Hábito } \\
\text { tabáquico } \\
(\%)\end{array}$} & No fumador & 48,7 & 51,3 & \multirow{3}{*}{$<0,001$} \\
\hline & Ex fumador & 47,3 & 52,7 & \\
\hline & Fumador & 62,6 & 37,4 & \\
\hline \multirow{6}{*}{ Sedentarismo } & Sueño nocturno (h/día, semana) & $6,0(6,0-7,0)$ & $6,0(6,0-7,0)$ & 0,162 \\
\hline & Sueño nocturno (h/día, fin de semana) & $8,0(7,0-8,0)$ & $7,0(7,0-8,0)$ & 0,001 \\
\hline & Siesta (h/día, fin de semana) & $0,0(0,0-0,5)$ & $0,0(0,0-0,5)$ & 0,429 \\
\hline & Tiempo de TV (h/día, semana) & $2,0(1,0-2,0)$ & $1,0(0,5-2,0)$ & $<0,001$ \\
\hline & Tiempo de TV (h/día, fin de semana) & $3,0(2,0-4,0)$ & $2,0(1,0-3,0)$ & $<0,001$ \\
\hline & Tiempo sentado (h/día, fin de semana) & $7,0(6,0-9,0)$ & $6,0(5,0-8,0)$ & $<0,001$ \\
\hline \multirow{7}{*}{$\begin{array}{l}\text { Características } \\
\text { de la dieta }\end{array}$} & Energía total (kcal/día) & $2864,7(2402,9-3366,3)$ & $2787,5(2298,8-3263,7)$ & 0,001 \\
\hline & Sodio (mg/día) & $3157,4(2500,8-4019,9)$ & $3004,8(2358,7-3787,9)$ & $<0,001$ \\
\hline & Fibra total (g/día) & $23,6(19,3-28,3)$ & $25,0(20,7-30,2)$ & $<0,001$ \\
\hline & Fibra insoluble (g/día) & $16,4(13,4-19,9)$ & $17,4(14,4-21,1)$ & $<0,001$ \\
\hline & Fibra soluble (g/día) & $7,1(5,7-8,6)$ & $7,6(6,1-9,1)$ & $<0,001$ \\
\hline & Colesterol dietético (mg/día) & $459,0(379,6-547,6)$ & $440,0(362,0-535,9)$ & 0,008 \\
\hline & Índice aMED & $3,64(1,69)$ & $4,22(1,75)$ & $<0,001$ \\
\hline
\end{tabular}

\begin{tabular}{|c|c|c|c|c|c|c|}
\hline \multicolumn{7}{|c|}{$\begin{array}{c}\text { Tabla } 4 \\
\text { Asociación entre el número de FRCV y la probabilidad de alcanzar } \\
\text { la adherencia a las recomendaciones de AF establecidas } \\
\text { por la Organización Mundial de la Salud }\end{array}$} \\
\hline & 0 (ref) & 1 & 2 & 3 & 4 & \multirow[b]{2}{*}{$\mathrm{p}$} \\
\hline & $\mathrm{n}=474$ & $\mathrm{n}=850$ & $\mathrm{n}=698$ & $\mathrm{n}=332$ & $\mathrm{n}=99$ & \\
\hline $\begin{array}{l}\text { OR Modelo crudo } \\
\quad(95 \% \mathrm{CI})\end{array}$ & 1,00 (ref) & $\begin{array}{c}0,60 \\
(0,48-0,76)\end{array}$ & $\begin{array}{c}0,47 \\
(0,37-0,59)\end{array}$ & $\begin{array}{c}0,33 \\
(0,24-0,44)\end{array}$ & $\begin{array}{c}0,18 \\
(0,11-0,29)\end{array}$ & $<0,001$ \\
\hline $\begin{array}{l}\text { OR Modelo } 1 \\
(95 \% \mathrm{CI})\end{array}$ & 1,00 (ref) & $\begin{array}{c}0,61 \\
(0,49-0,77)\end{array}$ & $\begin{array}{c}0,48 \\
(0,37-0,60)\end{array}$ & $\begin{array}{c}0,33 \\
(0,25-0,45)\end{array}$ & $\begin{array}{c}0,18 \\
(0,11-0,30)\end{array}$ & $<0,001$ \\
\hline $\begin{array}{l}\text { OR Modelo } 2 \\
(95 \% \mathrm{CI})\end{array}$ & 1,00 (ref) & $\begin{array}{c}0,62 \\
(0,49-0,78)\end{array}$ & $\begin{array}{c}0,49 \\
(0,38-0,62)\end{array}$ & $\begin{array}{c}0,34 \\
(0,25-0,46)\end{array}$ & $\begin{array}{c}0,19 \\
(0,11-0,32)\end{array}$ & $<0,001$ \\
\hline
\end{tabular}


Figura 1

Odds ratio para la adherencia a las recomendaciones de actividad física según el número de FRCV asociados (modelo 2)

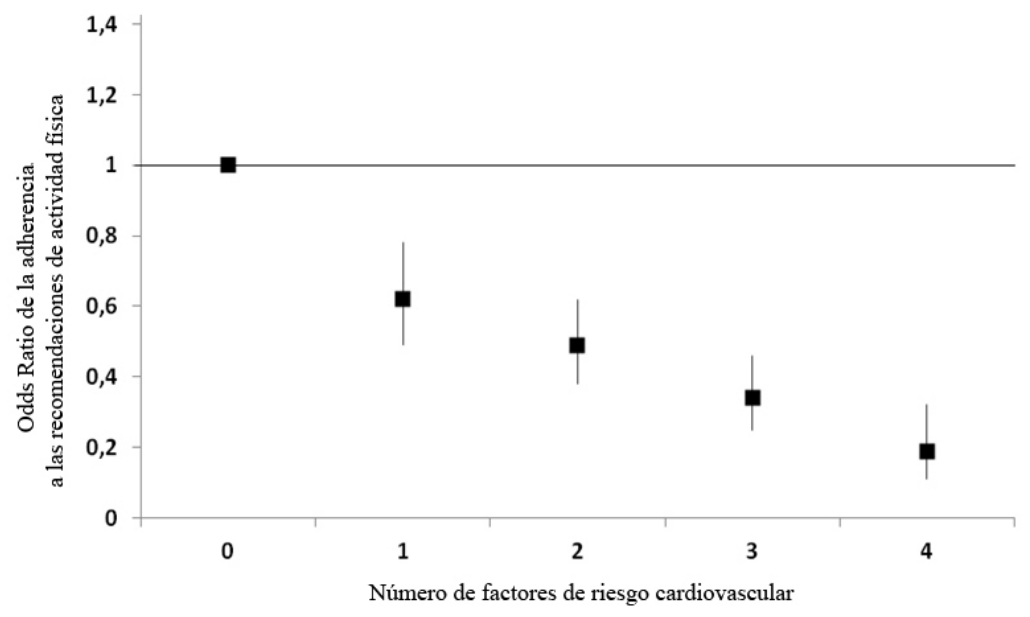

dL) $(\mathrm{p}<0,001)$, apolipoproteina B100 $(106,0$ vs $102,0 \mathrm{mg} / \mathrm{dL})(\mathrm{p}=0,024)$, cHDL $(51,0 \mathrm{vs}$ $54,0 \mathrm{mg} / \mathrm{dL})(\mathrm{p}<0,001)$ y apolipoproteina $\mathrm{A} 1$ $(140,0$ vs 146,0 mg/dL) $(\mathrm{p}<0,001)$.

Asimismo se hallaron diferencias en los niveles de glucosa en ayunas (96,0 vs 95,0 $\mathrm{mg} / \mathrm{dL}) \quad(\mathrm{p}=0,003), \mathrm{HbA} 1 \mathrm{c}(5,5$ vs $5,4 \%)$ $(\mathrm{p}<0,001)$, insulina $(7,0$ vs $5,6 \mathrm{uU} / \mathrm{mL})$ $(\mathrm{p}<0,001)$ e índice HOMA-IR $(1,68$ vs. 1,33$)$ $(\mathrm{p}<0,001)$, así como en el parámetro de inflamación PCR $(0,22$ vs $0,16 \mathrm{mg} / \mathrm{dL})(\mathrm{p}<0,001)$.

En los sujetos diagnosticados y en tratamiento por hipertensión arterial la prevalencia de adherencia a las recomendaciones de AF fue del 39,7\% $(\mathrm{p}<0,001)$, en el caso de las personas con dislipemia fue del $38,7 \%$ $(\mathrm{p}<0,001)$ y para quienes tenían diabetes mellitus fue del $32,7 \%(p=0,004)$.

La tabla 3 recoge las características de los estilo de vida que se asociaron al cumplimiento o no de las recomendaciones de AF. Con respecto al tabaquismo, las cifras de prevalencia fueron del $52,7 \%$ en exfumadores, $51,3 \%$ en no fumadores y $37,4 \%$ en fumadores $(\mathrm{p}<0,001)$.
En cuanto al sedentarismo, hubo diferencias estadísticamente significativas entre el grupo que sí seguía las recomendaciones y el que no las seguía, con respecto a horas de sueño nocturno en fin de semana $(8,0 v s$ 7,0 h/ día) $(\mathrm{p}=0,001)$, horas de televisión tanto entre semana $(2,0$ vs. 1,0 h/día $)(p<0,001)$ como en fin de semana $(3,0$ vs 2,0 h/día) $(\mathrm{p}<0,001)$ y tiempo sentado durante el fin de semana $(7,0$ vs 6,0 h/día) $(\mathrm{p}<0,001)$.

En relación a las características de la dieta se observaron diferencias significativas entre ambos grupos en ingesta total de energía $(2864,7$ vs $2787,5 \mathrm{kcal} /$ día $)(\mathrm{p}=0,001)$, sodio $(3157,4$ vs 3004,8 mg/día) $(\mathrm{p}<0,001)$, colesterol dietético (459,0 vs 440,0 mg/día) $(\mathrm{p}=0,008)$, consumo de fibra total $(23,6$ vs. $25,0 \mathrm{~g} /$ día $)(\mathrm{p}<0,001)$, soluble $(7,1$ vs $7,6 \mathrm{~g} /$ día) $(\mathrm{p}<0,001)$ e insoluble $(16,4$ vs 17,4 g/día $)$ $(\mathrm{p}<0,001)$ y en la adherencia al índice de calidad de dieta aMED $(3,64$ vs 4,22) $(\mathrm{p}<0,001)$.

En la tabla 4 se presentan los resultados del modelo multivariante de regresión logística que muestra la asociación entre la probabilidad de cumplir o no las recomendaciones de AF y el número de FRCV presentes en el sujeto. El modelo final ajustado por edad, sexo, 
nivel educativo y turno de trabajo (modelo 2): para un FRCV los resultados fueron OR: 0,62; 95\%CI: 0,49-0,78). Para dos factores OR: 0,$49 ; 95 \% \mathrm{CI}: 0,38-0,62)$, para tres OR: 0,34; 95\%CI: 0,25-0,46 y para cuatro FRCV OR: 0,19; 95\%CI: 0,11-0,32) (figura 1).

\section{DISCUSIÓN}

El presente trabajo muestra como menos de la mitad de la población estudiada cumple con las recomendaciones de AF elaboradas por la OMS. La adherencia es más frecuente en los trabajadores más jóvenes, con estudios superiores, trabajo de oficina y turno fijo diurno y en quienes adoptan estilos de vida más saludables: no son fumadores activos, son menos sedentarios sobretodo durante el tiempo de ocio y tienen una mayor adherencia a la dieta mediterránea. El seguimiento de estas recomendaciones es claramente inferior en el caso de presentar factores de riesgo cardiovascular.

Estudios previos desarrollados en población española presentan resultados muy diferentes, indicado una adherencia superior a la encontrada en nuestra investigación, con cifras de seguimiento del $75,8 \%$ o el $80,0 \%{ }^{15,16}$. Estas mismas discrepancias las encontramos en los análisis realizados en el resto del mundo. Una investigación llevada a cabo en veinte países para valorar las cifras de adecuación a las recomendaciones mostró una gran variabilidad en función del territorio estudiado, con cifras del 56,6\% en Japón, 57,0\% en Bélgica, $73,8 \%$ en Portugal, $84,1 \%$ en EEUU o el 93, $2 \%$ en China ${ }^{15}$.

La comparación entre estudios resulta en ocasiones compleja debido a la variabilidad en el diseño y la metodología aplicada. No todas las guías utilizadas como referencia para valorar la adherencia tienen en cuenta las actividades desarrolladas en el trabajo, en desplazamientos o en el desempeño de tareas domésticas. Además, no en todos los estudios se registra la AF llevada a cabo durante el mismo periodo de tiempo. En nuestro análisis se evaluó el efecto de la AF desarrollada a lo largo de un año, por lo que los efectos observados en los parámetros clínicos creemos que podrían ser más representativos que en los que solo se reportó la AF llevada a cabo durante la última semana. El tipo de cuestionario utilizado o el uso de medidas objetivas, como los acelerómetros, pueden hacer variar los resultados. Algunos cuestionarios sobreestiman la AF, sobretodo la moderada ${ }^{17,18}$. Se ha demostrado además que con medidas objetivas el porcentaje de individuos que siguen las recomendaciones es muy inferior ${ }^{19,20}$. No obstante, los cuestionarios suelen resultar muy útiles para valorar la AF en estudios epidemiológicos con grandes muestras. Así mismo, la práctica de AF disminuye con la edad, y el porcentaje de individuos que reportan no realizar ningún tipo de AF se incrementa, motivo por el que los resultados derivados de nuestro análisis podrían reflejar una adherencia inferior a otros llevados a cabo en España en población con un rango de edad mucho más amplio.

El grado de seguimiento de las recomendaciones encontrado en la presente muestra se redujo considerablemente en los personas diagnosticadas y tratadas por diferentes FRCV. Además, este conjunto de trabajadores había recibido consejo no pautado sobre la práctica de $\mathrm{AF}$.

Desde hace tiempo, diferentes resultados refrendan los efectos beneficiosos que la práctica de al menos 150 minutos semanales de AF moderada o intensa tiene sobre la salud cardiorespiratoria y metabólica ${ }^{21}$. Además, la disminución de peso que junto con otras modificaciones higiénico-dietéticas, como la reducción de la ingesta calórica, lleva asociada la práctica regular de AF, mejora el perfil lipídico y afecta favorablemente a otros parámetros de riesgo cardiovascular a menudo presentes en individuos dislipémicos ${ }^{22}$.

El presente análisis se llevó a cabo sobre una amplia muestra que podría ser representativa de la población general masculina de entre 40 y 55 años y estatus socioeconómico, pero que al ser transversal no nos permite es- 
tablecer una relación de causa efecto. Observamos un perfil metabólico claramente desfavorable en los trabajadores que no siguen las recomendaciones, con mayor dislipemia, obesidad, diabetes mellitus e hipertensión e incluso con parámetros inflamatorios y de resistencia a insulina más elevados. No podemos saber, ni era el objetivo del presente trabajo, si la escasa AF realizada trae como consecuencia ese perfil o es la presencia de obesidad, hipertensión o dislipemia la que dificulta el seguimiento de estas recomendaciones. Sin embargo, resulta llamativo que precisamente los trabajadores que siguen menos las recomendaciones sean aquellos en quienes están más indicadas y que cuantos más FRCV se asocien menor seguimiento se efectúa.

A pesar de la evidencia científica descrita anteriormente sobre los beneficios de la AF, los resultados reportados en un reciente estudio llevado a cabo en nueve países europeos muestran como en las consultas de atención primaria son más los consejos dirigidos a la modificación de otros componentes del estilo de vida que a la promoción y orientación en la práctica de $\mathrm{AF}$ en pacientes con alto riesgo de $\mathrm{ECV}^{23}$, además de haber una mayor documentación y registro de FRCV clásicos como hipertensión, diabetes mellitus y dislipemia frente a FRCV asociados a la conducta como el sedentarismo o el tabaquismo ${ }^{23}$. Los motivos declarados por los trabajadores de atención primaria para explicar esta situación son diversos. Dudan de la efectividad de informar, no se sienten cómodos dando un consejo detallado sobre la realización de AF y además esgrimen falta de tiempo, por lo que muchos sanitarios adoptan como medida terapéutica prioritaria la administración de fármacos ${ }^{24}$.

Nuestros resultados muestran que el grado de adherencia es mayor en las personas que tienen estudios superiores y desarrollan trabajos administrativos frente a quienes cuentan con un nivel educativo inferior y desarrollan trabajos manuales aunque de intensidad ligera. Dada la importante contribución que supone la actividad ocupacional al conjunto de la AF llevada a cabo por una persona a lo largo del día, puede resultar llamativo que los sujetos que realizan trabajos de oficina presenten una prevalencia mayor en el cumplimiento de las recomendaciones de AF. No obstante, el hecho de ser trabajadores manuales no implica que en todos los casos se lleve a cabo una actividad de intensidad moderada o vigorosa en el desempeño de la labor profesional, que puede consistir en estar de pie la mayor parte de la jornada laboral sin realizar grandes esfuerzos ni desplazamientos. Además, los resultados revelan como el turno fijo, al que corresponden la práctica totalidad de los trabajadores de oficina, favorece la adherencia a las recomendaciones, probablemente debido a una mayor facilidad para conciliar la jornada laboral con el tiempo de ocio, lo que facilita la realización de AF frente a quienes varían de turno de trabajo cada semana, para quienes es más difícil desarrollar el hábito de la práctica de AF durante el tiempo libre.

Es previsible que en los próximos años aumenten las enfermedades condicionadas por el exceso de peso como la hipertensión, la diabetes mellitus o la dislipemia. La AF es un pilar fundamental en el tratamiento, por ello, es absolutamente necesario buscar fórmulas que logren su incremento a nivel poblacional, pero sobre todo en quienes ya presentan algún FRCV. La mera información parece no ser suficiente e incluso derivar esta responsabilidad únicamente a los profesionales de la sanidad no resulta efectivo por lo que es necesario un esfuerzo imaginativo que implique a varios agentes sociales. Son muy interesantes las experiencias recientes que intentan facilitar al máximo esta actividad, incluyendo en el entorno de trabajo el acceso a los gimnasios u organizando grupos pequeños con la idea de apoyarse mutuamente para lograr incrementos de actividad, dentro del marco de la prevención de riesgos laborales y la responsabilidad social corporativa. 
El estudio presenta las limitaciones propias del uso de una herramienta no objetiva como son los cuestionarios, pese al hecho de haber sido validados con acelerómetros y disponer de personal entrenado para llevar a cabo las entrevistas. Además, como se ha indicado con anterioridad, el hecho de ser un estudio transversal no permite establecer causalidad. Asimismo, esta muestra no es representativa de la población general, ya que por su condición de trabajadora podría existir un sesgo, al ser personas sanas o al menos sin enfermedades incapacitantes.

En conclusión, el nivel de seguimiento de las recomendaciones de actividad física en la población estudiada sigue siendo insuficiente, sobre todo en las personas que presentan mayor número de factores de riesgo cardiovascular. Por este motivo, es necesario trabajar en la promoción y difusión del mensaje para lograr un seguimiento efectivo de las recomendaciones llevadas a cabo en salud pública que, además de implicar un bajo costo, pueden representar un buen mecanismo de prevención y mejora de determinadas patologías muy prevalentes, como son las cardiovasculares.

\section{AGRADECIMIENTOS}

Agradecemos su colaboración a los participantes y al personal del estudio Aragon Workers Health Study.

\section{BIBLIOGRAFÍA}

1. World Health Organization. Global recommendations on physical activity for health. Ginebra: World Health Organization; 2010.

2. Garber CE, Blissmer B, Deschenes MR, Franklin BA, Lamonte MJ, Lee IM, et al. American College of Sports Medicine position stand. Quantity and quality of exercise for developing and maintaining cardiorespiratory, musculoskeletal, and neuromotor fitness in apparently healthy adults: guidance for prescribing exercise. Med Sci Sports Exerc. 2011; 43:1334-1359.

3. Eckel RH, Jakicic JM, Ard JD, de Jesus JM, Houston Miller N, Hubbard VS, et al. 2013 AHA/ACC guideline on lifestyle management to reduce cardiovascular risk: a report of the American College of Cardiology/American Heart Association Task Force on Practice Guidelines. J Am Coll Cardiol. 2014; 63:2960-2984.
4. World Health Organization. Global health risks: mortality and burden of disease attributable to selected major risks. Ginebra: World Health Organization; 2009.

5. Baker PR, Francis DP, Soares J, Weightman AL, Foster C. Community wide interventions for increasing physical activity. Cochrane Database Syst Rev. 2011; CD008366.

6. Casasnovas JA, Alcaide V, Civeira F, Guallar E, Ibanez B, Borreguero JJ, et al. Aragon workers' health study--design and cohort description. BMC Cardiovasc Disord. 2012; 12:45.

7. Martínez-González MA, López-Fontana C, Varo JJ, Sanchez-Villegas A, Martínez JA. Validation of the Spanish version of the physical activity questionnaire used in the Nurses' Health Study and the Health Professionals' Follow-up Study. Public Health Nutr. 2005; 8:920-927.

8. Martin-Moreno JM, Boyle P, Gorgojo L, Maisonneuve P, Fernandez-Rodriguez JC, Salvini S, et al. Development and validation of a food frequency questionnaire in Spain. Int J Epidemiol. 1993; 22:512-519.

9. de la Fuente-Arrillaga C, Ruiz ZV, Bes-Rastrollo M, Sampson L, Martinez-Gonzalez MA. Reproducibility of an FFQ validated in Spain. Public Health Nutr. 2010; 13:1364-1372.

10. Moreiras O, Carbajal A, Cabrera L, Cuadrado C. Tablas de composicion de alimentos. 15 ed. Madrid: Ediciones Pirámide; 2011.

11. Mataix Verdú J. Tabla de composición de alimentos. 5 ed. Granada: Universidad de Granada; 2009.

12. Fung TT, Rexrode KM, Mantzoros CS, Manson JE, Willett WC, Hu FB. Mediterranean diet and incidence of and mortality from coronary heart disease and stroke in women. Circulation. 2009; 119:10931100 .

13. Friedewald WT, Levy RI, Fredrickson DS. Estimation of the concentration of low-density lipoprotein cholesterol in plasma, without use of the preparative ultracentrifuge. Clin Chem. 1972; 18:499-502.

14. Matthews DR, Hosker JP, Rudenski AS, Naylor BA, Treacher DF, Turner RC. Homeostasis model assessment: insulin resistance and beta-cell function from fasting plasma glucose and insulin concentrations in man. Diabetologia. 1985; 28:412-419.

15. Bauman A, Bull F, Chey T, Craig CL, Ainsworth BE, Sallis JF, et al. The International Prevalence Study on Physical Activity: results from 20 countries. Int J Behav Nutr Phys Act. 2009; 6:21. 
16. Rodriguez-Romo G, Cordente CA, Mayorga JI, Garrido-Muñoz M, Macias R, Lucia A et al. Influencia de determinantes socio-demográficos en la adherencia a las recomendaciones de actividad física en personas de entre 15 y 74 años de Madrid. Rev Esp Salud Publica. 2011; 85:351-362.

17. Lee PH, Macfarlane DJ, Lam TH, Stewart SM. Validity of the International Physical Activity Questionnaire Short Form (IPAQ-SF): a systematic review. Int J Behav Nutr Phys Act. 2011; 8:115.

18. Ekelund U, Sepp H, Brage S, Becker W, Jakes R, Hennings $\mathrm{M}$, et al. Criterion-related validity of the last 7-day, short form of the International Physical Activity Questionnaire in Swedish adults. Public Health Nutr. 2006; 9:258-265.

19. Tucker JM, Welk GJ, Beyler NK. Physical activity in U.S.: adults compliance with the Physical Activity Guidelines for Americans. Am J Prev Med. 2011; 40:454461.

20. Troiano RP, Berrigan D, Dodd KW, Masse LC, Tilert T, McDowell M. Physical activity in the United States measured by accelerometer. Med Sci Sports Exerc. 2008; 40:181-188.

21. Physical Activity Guidelines Advisory Committee report, 2008. To the Secretary of Health and Human Services. Part A: executive summary. Nutr Rev. 2009; 67:114-120

22. Reiner Z, Catapano AL, De Backer G, Graham I, Taskinen MR, Wiklund O, et al. ESC/EAS Guidelines for the management of dyslipidaemias: the Task Force for the management of dyslipidaemias of the European Society of Cardiology (ESC) and the European Atherosclerosis Society (EAS). Eur Heart J. 2011; 32:1769-1818.

23. Ludt S, Petek D, Laux G, van Lieshout J, Campbell SM, Kunzi B, et al. Recording of risk-factors and lifestyle counselling in patients at high risk for cardiovascular diseases in European primary care. Eur J Prev Cardiol. 2012; 19:258-266.

24. Hebert ET, Caughy MO, Shuval K. Primary care providers' perceptions of physical activity counselling in a clinical setting: a systematic review. Br J Sports Med. 2012; 46:625-631. 